\title{
Discrete Fractional-Order Systems with Applications in Engineering and Natural Sciences
}

\author{
Abdelalim Elsadany $\mathbb{D}^{1},{ }^{1}$ Abdulrahman Al-khedhairi $\mathbb{D}^{2},{ }^{2}$ Hamdy Nabih Agiza, ${ }^{3}$ \\ Baogui Xin (D), ${ }^{4}$ and Amr Elsonbaty ${ }^{5}$ \\ ${ }^{1}$ Suez Canal University, Ismailia, Egypt \\ ${ }^{2}$ King Saud University, Riyadh, Saudi Arabia \\ ${ }^{3}$ Mansoura University, Mansoura, Egypt \\ ${ }^{4}$ Shandong University of Science and Technology, Qingdao, China \\ ${ }^{5}$ Prince Sattam Bin Abdulaziz University, Al-Kharj, Saudi Arabia \\ Correspondence should be addressed to Abdelalim Elsadany; aelsadany1@yahoo.com
}

Received 5 January 2022; Accepted 5 January 2022; Published 27 January 2022

Copyright (c) 2022 Abdelalim Elsadany et al. This is an open access article distributed under the Creative Commons Attribution License, which permits unrestricted use, distribution, and reproduction in any medium, provided the original work is properly cited.

Discrete fractional calculus (DFC) research is gaining a lot of attention, both theoretically and practically. Some DFC systems such as the discrete fractional-order logistic, the fractional-order Henon maps, and their applications in image encryption have been studied. Recent publications in this field include the novel discrete fractional order in engineering, physical, biological, and economical models, analytical insights into such models, stability analysis, bifurcation analysis, determination of chaotic behaviour, and implementation of chaos control methods and synchronization applications in many applied models.

We are delighted to announce the publication of this Special Issue devoted to fresh problems in discrete fractional calculus (DFC) and its applications in engineering and natural sciences. The main goal of this Special Issue is to provide an opportunity to study new developments in the discrete fractional-order models in relevant areas of engineering and natural sciences, as well as bifurcation and chaos analysis of discrete fractional-order models and their applications. Our editorial team chose ten pieces for publishing from among those submitted for consideration. These articles cover the topics of feedback controller for fractional-order T-S fuzzy system, delta partial difference equations, forecasting confirmed cases, deaths, and recoveries from COVID-19 in China, discrete fractionalorder Prion model, $\mathrm{n}$-dimensional fractional frequency Laplace transform, fractional Black-Scholes model, impulsive problem under Caputo fractional boundary conditions, discrete-time fractional-order system and its hidden chaotic attractors, algorithm for R-L fractional nonlinear control systems, and social welfare of a two-stage game under R\&D spillovers. We are hopeful that this Special Issue will contribute to a better understanding and research of discrete fractional calculus and its applications. 


\section{Conflicts of Interest}

The Guest Editors declare that they have no conflicts of interest regarding the publication of this Special Issue.

Abdelalim Elsadany Abdulrahman Al-khedhairi Hamdy Nabih Agiza

Baogui Xin Amr Elsonbaty 